\title{
LoRa Base-Station-to-Body Communication with SIMO Front-to-Back Diversity
}

\author{
Thomas Ameloot, Patrick Van Torre and Hendrik Rogier, Senior Member, IEEE
}

\begin{abstract}
The LoRa standard is currently widely employed for low-power long-range wireless sensor networks at sub-GHz frequency bands. The longer wavelengths associated with subGHz technology provide excellent radiowave propagation characteristics, yielding much larger coverage compared to higher frequency bands. In the case of wearable sensors, the $868 \mathrm{MHz}$ band can be covered by textile substrate-integrated-waveguide antennas of a convenient size. In body-centric communication systems, front-to-back diversity is an important asset to mitigate the shadowing of the antennas by the presence of the human body. This paper describes a diversity textile-antenna-based LoRa platform with integrated transceivers. Outdoor measurement campaigns are conducted to assess the performance of the wearable LoRa nodes with front-to-back diversity in an urban radio propagation environment at walking and cycling speeds. These experiments prove that large ranges of $1.5 \mathrm{~km}$ can easily and reliably be achieved for off-body LoRa communication links. The results demonstrate a significant performance improvement in terms of packet loss in NLoS situations when comparing single-receiver performance to different spacial receiver diversity applications. Additionally, link budget increases up to $5.5 \mathrm{~dB}$ are seen, owing to the realized diversity gain.
\end{abstract}

Index Terms-Body-centric communication, Diversity, LoRa, Substrate Integrated Waveguide, Textile Antenna

\section{INTRODUCTION}

Wireless sensor networks are widely employed for a plethora of data links operating in the industrial, scientific and medical (ISM) bands. ISM-band systems have the advantage of operating in unlicensed parts of the spectrum, allowing private networks on a free-to-air basis. For these networks, the most widely used system is $\mathrm{WiFi}$, which operates at $2.45 \mathrm{GHz}$ or $5.8 \mathrm{GHz}$ frequencies. These bands offer a wide bandwidth but also suffer from a limited range due to the difficult radiowave propagation conditions in indoor environments, with large attenuation caused by walls and other structures. More narrowband systems such as IEEE 802.15.4 (physical layer for ZigBee) allow for an extension of the range, which is however still limited.

In contrast, sub-GHz frequencies provide more favorable propagation characteristics, with signal penetration through building structures with limited attenuation. In Europe, two sub-GHz ISM bands are currently available, at $434 \mathrm{MHz}$ and $868 \mathrm{MHz}$. Whereas the lowest frequency band offers the best radio propagation characteristics, suitable antennas tend

The authors are with Ghent University - imec, IDLab, Department of Information Technology (INTEC), Technologiepark-Zwijnaarde 15, 9052 Gent, Belgium (email: thomas.ameloot@ugent.be)

This work was partly funded by the Research Foundation - Flanders (FWO) through the "MUlti-SErvice WIreless NETwork", FWO-FRS Excellence of Science - EOS project. to be rather large, due to the wavelength of about $70 \mathrm{~cm}$. At $868 \mathrm{MHz}$, the wavelength and, hence, also the antennas are only half that size, but the propagation characteristics are still considerably better than at $2.45 \mathrm{GHz}$ or higher. By employing substrate-integrated-waveguide (SIW) technology, efficient and compact wearable textile antennas have been developed for the $868 \mathrm{MHz}$ band [1]-[3]. In that band, LoRa technology [4] has been proven to offer communication ranges of over $10 \mathrm{~km}$ in outdoor environments [5], [6]. Recently, a compact LoRa node with extended dynamic range for channel measurement purposes has been documented in [7]. The integration of this type of node on an SIW antenna results in a wearable unit for off-body LoRa communication, able to cover kilometer range links [8]. In this paper, we investigate the performance of a novel body-worn LoRa frontto-back diversity wireless communication system, based on these recent developments.

Using LoRa for off-body communication is not a completely new concept. [9], [10] and [11] describe LoRa systems that monitor vital signs and environmental data while worn on the body. Unfortunately, these publications do not discuss the performance of the wireless links besides mentioning the available communication ranges. In contrast, [12], [13] and [14] present the deployment of an arm-mounted LoRa platform to more rigorously evaluate the feasibility of applying LoRa in a bodycentric wireless communication system. In [12] and [13], the nodes are deployed in an indoor environment to assess the application of LoRa for health and wellbeing monitoring. In [14], the results from an outdoor measurement campaign are presented and analyzed to characterize the wireless channel. Similar to this paper, [12], [13] and [14] concern base-stationto-body communication. However, there are a few decisive differences between these publications and the work presented here. All three of these earlier publications present data that were gathered over a longer time interval, whereas this work presents a real-time link monitoring effort during which the test person is following a fixed trajectory. Consequently, this contribution focuses more on real-time link performance and achieves a much finer spatial resolution. Additionally, in [12], [13] and [14], commercial LoRaMote devices were used to gather data, whereas in this work, custom LoRa channel characterization hardware was deployed that dynamically implements different dynamic range settings. Moreover, by using textile materials, the SIW antennas in this system are very light and bendable. These textile antennas are deployed on the front and back of the torso of the test person, as this placement is more robust against depolarization for dynamic users [15]. Yet, the biggest advancement made in this contribution with 
TABLE I: Comparison of recent literature on the application of LoRa in body-centric wireless networks.

\begin{tabular}{|c|c|c|c|c|c|}
\cline { 2 - 6 } \multicolumn{1}{c|}{} & [8] & {$[12]$} & {$[13]$} & {$[14]$} & This work \\
\hline $\begin{array}{c}\text { Environment } \\
\text { strategy }\end{array}$ & $\begin{array}{c}\text { Communication } \\
\text { range test }\end{array}$ & $\begin{array}{c}\text { Obtaining channel } \\
\text { statistics }\end{array}$ & $\begin{array}{c}\text { Obtaining channel } \\
\text { statistics }\end{array}$ & $\begin{array}{c}\text { Obtaining channel } \\
\text { statistics }\end{array}$ & Real-time link monitoring \\
\hline $\begin{array}{c}\text { Measurement } \\
\text { hardware }\end{array}$ & $\begin{array}{c}\text { Custom channel } \\
\text { characterization } \\
\text { device integrated on } \\
\text { textile antenna }\end{array}$ & $\begin{array}{c}\text { Commercial } \\
\text { LoRaMote device } \\
\text { with PCB antenna }\end{array}$ & $\begin{array}{c}\text { Commercial } \\
\text { LoRaMote device } \\
\text { with PCB antenna }\end{array}$ & $\begin{array}{c}\text { Commercial } \\
\text { LoRaMote device } \\
\text { with PCB antenna }\end{array}$ & $\begin{array}{c}\text { Custom channel } \\
\text { characterization device } \\
\text { integrated on textile antenna }\end{array}$ \\
\hline Wireless link & Body-to-body & Body-to-base-station & Body-to-base-station & Body-to-base-station & Body-to-base-station \\
\hline $\begin{array}{c}\text { Antenna } \\
\text { placement }\end{array}$ & $\begin{array}{c}\text { Worn on front of } \\
\text { torso }\end{array}$ & Worn on arm & Worn on arm & Worn on arm & $\begin{array}{c}\text { Worn on front and back of } \\
\text { torso }\end{array}$ \\
\hline $\begin{array}{c}\text { Diversity } \\
\text { techniques }\end{array}$ & None & None & None & $\begin{array}{c}\text { Multiple-Output (SIMO) } \\
\text { Selection Combining \& } \\
\text { Maximum-Ratio Combining }\end{array}$ \\
\hline Focus on... & Measurement & $\begin{array}{c}\text { Use of different } \\
\text { carrier frequency } \\
\text { channels }\end{array}$ & $\begin{array}{c}\text { Use of different } \\
\text { carrier frequency } \\
\text { channels and } \\
\text { modulation settings }\end{array}$ & Nakagami model \\
fitting & $\begin{array}{c}\text { Influence of using different } \\
\text { types of diversity and effect } \\
\text { of different mobile user } \\
\text { velocities }\end{array}$ \\
\hline
\end{tabular}

respect to previous research is undoubtedly the application of receiver diversity and additionally, the investigation of link quality at higher speeds. In the following paragraphs, both concepts are briefly introduced. A summarized comparison between the most relevant existing literature and this work is given in Table I.

Although a single LoRa unit is definitely able to transmit and receive data over a large range, in off-body communication systems where wearable, low-profile antennas are deployed, the communication link often suffers from shadowing by the human body [14], [16], [17]. As demonstrated extensively by many researchers for the $2.45 \mathrm{GHz}$ band, front-to-back (F/B) diversity is a key asset for improving the reliability of the wireless link [18]. The performance of diversity antenna systems worn on the front and back of the torso have been documented for indoor environments at $868 \mathrm{MHz}$ in [19] and [20], and at $2.45 \mathrm{GHz}$ in [21], [22] and [23]. At sub-GHz frequencies, diffraction of waves around the body is more present. However, significant body shadowing still exists and additionally, the wearable antennas themselves have a directional radiation pattern, approximately covering a hemisphere around the body. Therefore, front-to-back diversity systems are expected to remain useful at sub-GHz frequency bands, enabling significantly more robust off-body communication over larger distances.

From the viewpoint of the mobile user, Doppler spread also has an important impact on the wireless link quality. Earlier research has concluded that the LoRa modulation protocol is fairly Doppler-resistant [24], [25]. Other research states that for LoRa, the $868 \mathrm{MHz}$ channel is sufficiently reliable at low speeds (up to $25 \mathrm{~km} / \mathrm{h}$ ), but performance is seen to deteriorate for speeds upwards of $40 \mathrm{~km} / \mathrm{h}$ [26]. This work compares the link quality measured at walking speeds and at an average speed of $31.1 \mathrm{~km} / \mathrm{h}$, which is considered a good approximation of the maximum speed of an average cyclist. Additionally, it is seen as a credible upper bound for the speed of alternative personal transport vehicles such as motorized steps, unicycles or skateboards. To comfortably reach higher speeds, a mobile user would need to use a different means of transport, such as a motorcycle or car. In these cases, one might no longer need to use battery-powered, body-worn nodes for data communication.

As mentioned earlier, this work investigates the performance of a body-worn LoRa front-to-back diversity communication system. The results are based on two outdoor base-station-tobody measurement campaigns at $868 \mathrm{MHz}$. In the first measurement campaign, the improvement achieved by applying $\mathrm{F} / \mathrm{B}$ diversity is examined, whilst in the other measurement campaign the influence of the speed of the user on the performance of the links is investigated. The paper is further organized as follows. In Section II, the measurement infrastructure and methodology are described. The measurement results are presented and analyzed in detail in Section III. Finally, a conclusion completes the paper in Section IV.

\section{Materials And Methods}

\section{A. LoRa base station}

A fixed, battery-powered LoRa transmitter is placed on top of a $57 \mathrm{~m}$ high building in an urban environment (Fig. 1). The transmitter has a maximum output power of $10 \mathrm{dBm}$ and is connected via a $50 \Omega$ coaxial cable to a a wire-based groundplane monopole antenna. This transmitter sends ten 1-byte packets per second, including only an incrementing packet number. The very short packet length guarantees the
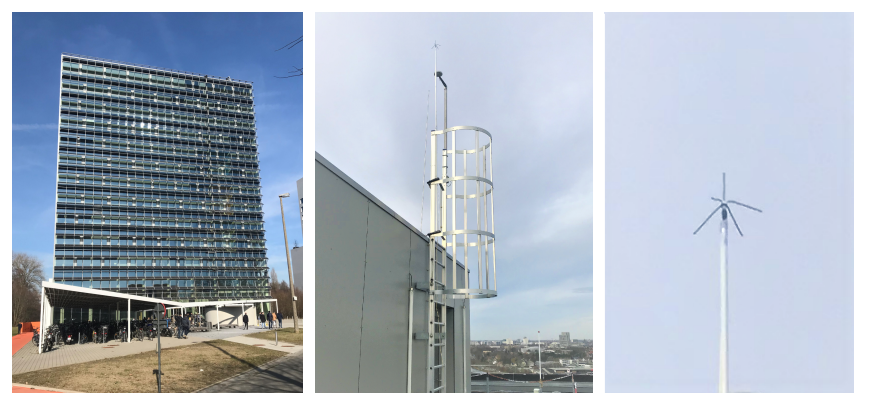

Fig. 1: Base station antenna (right) on top of the access ladder (middle) to the uppermost roof portion of the office building (left) located at marker A. 
shortest possible airtime per transmission and allows to transmit at this fairly high repetition rate. The power output of the LoRa hardware connected to the omnidirectional base-station antenna was calibrated using a Rohde \& Schwartz (R\&S) FSV40 spectrum analyzer.

\section{B. Wearable LoRa system}

The body-worn LoRa system consists of two identical nodes, which are displayed in Fig. 2, worn on the front and back of the body to implement diversity. These units consist of a textile SIW antenna [1] on which a compact LoRa transceiver is integrated, along with a low-power microcontroller, $32 \mathrm{Mbit}$ of flash memory and a triaxial magnetometer, accelerometer and gyroscope. The unit is also equipped with a very lowprofile battery, preserving the compactness and wearability of the node. In Fig. 3, the normalized directivity of the standalone textile SIW antenna is compared to that of the entire system when worn on the body. Additional hardware design features and operational principles of this transceiver node are broadly discussed in [7] and [8].
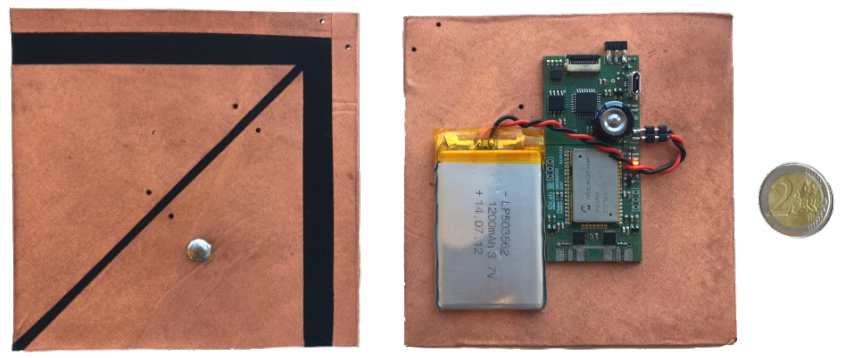

Fig. 2: Front (left) and back (right) side of the wearable LoRa unit, with the complete system integrated on a textile SIW antenna.

Fig. 3 primarily shows that a sizable portion of the energy radiated along the backward hemisphere of the antenna is absorbed by the wearer. With the average front-to-back $(\mathrm{F} / \mathrm{B})$ ratio of the body-worn node equaling as much as $9.57 \mathrm{~dB}$, it can be concluded that body shadowing is in fact a very prominent link obstruction mechanism for the wearable system. Fig. 3 also shows that the directivity of the body-worn node is highly regular in front of the coronal plane. Given the large amount of small variations in the orientation and posture of the test person during the measurement campaigns, limited variations in the radiation pattern are assumed to be averaged out across a large range of angles when moving through the environment. A similar reasoning can be applied to the expected effects of the single notch on the back side of the radiation pattern as the cumulative impact of this notch is assumed to be distributed across the (already heavily attenuated) back hemisphere of the pattern when the test person is moving. In consideration of this mechanism and the high $\mathrm{F} / \mathrm{B}$ ratio presented earlier, in this paper, the analysis is based on the separate and combined results gathered from the front and back nodes, assuming half dome radiation patterns in front of the body with some leakage around the body. As a consequence, in addition to path loss, shadowing by the environment, small-scale fading, and reflections in the environment, body-centric link parameters such as body shadowing and variations in the orientation and posture of the test person are considered to be part of the physical link under study.

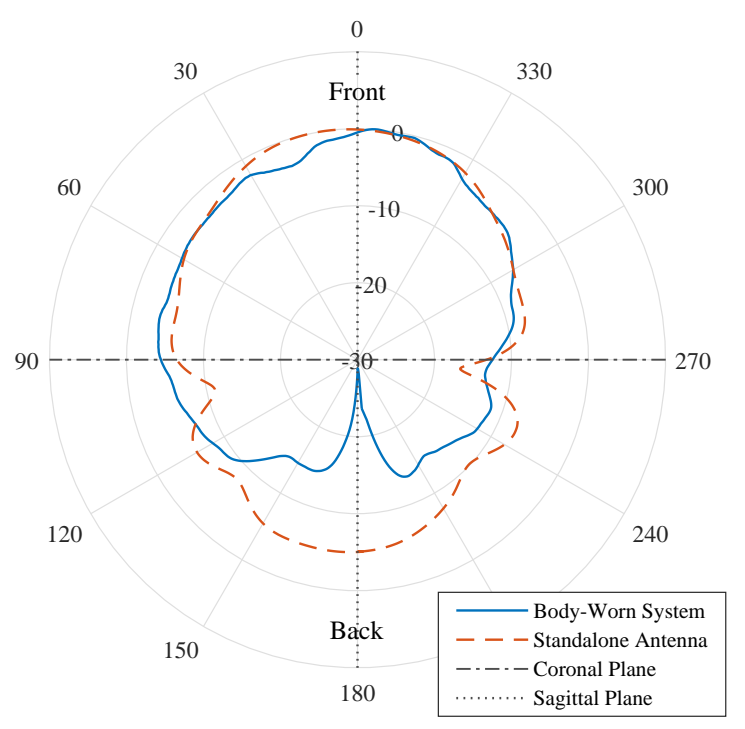

Fig. 3: Normalized directivity (in $\mathrm{dB}$ ) of the standalone textile SIW antenna and the fully integrated LoRa system when worn on the chest.

Power level measurements performed by this hardware are calibrated using a three-phase calibration procedure, which is comprehensively described in [7]. The first phase of this procedure consists of calibrating the power output of a reference node when directly connected to the R\&S FSV40 spectrum analyzer. Then, a high-precision stepped attenuator and a set of highly shielded coaxial cables are placed between the reference node and the spectrum analyzer. Next, this setup is calibrated for all possible attenuation settings. In the third phase, the reference node is used to inject a signal into the now calibrated stepped attenuator. Finally, the power levels measured by the device under test (DUT) can be compared to those measured in the previous phase to calibrate the receiver. To shield the DUT from any leakage from the reference node or the stepped attenuator, both components are placed in an anechoic chamber, while the DUT is installed in the shielded control room adjacent to this chamber.

\section{Measurement Strategy}

Due to their high system integration level, LoRa transceivers provide very few options to extract channel state information. Moreover, a very limited dynamic range in terms of signalto-noise ratio (SNR) is typically observed as strong signals tend to easily saturate the detector [5], [7]. In [7], this is circumvented by integrating stepped attenuators onto the wireless node. Their attenuation settings are based on the signal strength of previously received packets, enabling these nodes to adapt to different transmission path lengths and propagation losses. However, given the very rapid variations of the wireless 
channel between a fixed base station and a mobile receiver, this kind of adaptive scheme for extending the dynamic range is not suitable for the experiments presented in this work. Consequently, a different measurement strategy was adopted.

Keeping in mind that a high channel sampling rate is desirable when a mobile user is involved, a low LoRa spreading factor (SF) of 7 was chosen, which greatly limits the airtime needed for transmitting a single data packet. Unfortunately, using a lower SF also lowers the sensitivity of the system, which has an adverse effect on the already limited dynamic range, revealing a trade-off between the channel sounding dynamic range and sampling rate. In this case, the dynamic range was reduced to $15 \mathrm{~dB}$. However, as it has been demonstrated that attenuators can be used to shift the channel sounding dynamic range up [7], the increased packet transmission rate can be exploited to transmit packets at varying transmission powers in quick succession to probe the channel at different dynamic range settings. Varying the transmission power and transmitter attenuation settings enables the body-worn nodes to characterize the channel over a relatively large dynamic range of about $60 \mathrm{~dB}$, which is composed of four $15 \mathrm{~dB}$ subranges according to the predefined scheme, shown in Table II.

TABLE II: Dynamic range settings.

\begin{tabular}{|c|c|c|c|c|}
\hline Packet $\mathrm{n}^{\circ}$ mod 4 & 0 & 1 & 2 & 3 \\
\hline $\mathrm{P}_{\mathrm{TX}}$ & $10 \mathrm{dBm}$ & $5 \mathrm{dBm}$ & $0 \mathrm{dBm}$ & $-5 \mathrm{dBm}$ \\
$\mathrm{ATT}_{\mathrm{TX}}$ & $0 \mathrm{~dB}$ & $10 \mathrm{~dB}$ & $20 \mathrm{~dB}$ & $30 \mathrm{~dB}$ \\
\hline $\mathrm{P}_{\mathrm{TX}, \text { total }}$ & $10 \mathrm{dBm}$ & $-5 \mathrm{dBm}$ & $-20 \mathrm{dBm}$ & $-35 \mathrm{dBm}$ \\
$\mathrm{ATT}_{\mathrm{TX}, 10 \mathrm{dBm}}$ & $0 \mathrm{~dB}$ & $15 \mathrm{~dB}$ & $30 \mathrm{~dB}$ & $45 \mathrm{~dB}$ \\
\hline
\end{tabular}

This range could be extended further, to a theoretical maximum of $90 \mathrm{~dB}$, by also dynamically using the attenuators at the receiver side of the link. However, more packets would need to be sent to perform a single scan of this $90 \mathrm{~dB}$ dynamic range. As the highest power level received during preliminary measurements rarely exceeded half of the maximum power that can be measured by the system, this extension was not implemented. When receiving a data packet, the dynamic range settings used to transmit it can be found by considering the remainder of the division of the packet number by 4 .

Subtracting the transmitter attenuation $\mathrm{ATT}_{\mathrm{TX}}$ from the corresponding transmit power $\mathrm{P}_{\mathrm{TX}}$ yields the total transmission power $\mathrm{P}_{\mathrm{TX}}$,total for each of the four dynamic range settings. The power that would be received when always transmitting at $10 \mathrm{dBm}$ can eventually be described as the sum of the power level measured by the receiver and the virtual attenuation applied to the transmitter w.r.t. this constant transmit power of $10 \mathrm{dBm}\left(\mathrm{ATT}_{\mathrm{TX}, 10 \mathrm{dBm}}\right)$. Using this metric, the channel attenuation can be calculated as follows:

$$
\mathrm{ATT}_{\mathrm{CH}}=10 \mathrm{dBm}-\mathrm{P}_{\mathrm{RX}, \text { meas }}+\mathrm{ATT}_{\mathrm{TX}, 10 \mathrm{dBm}}
$$

in which $\mathrm{P}_{\mathrm{RX} \text {,meas }}$ denotes the power that was measured by the receiver. The measurement scheme presented in Table II was calibrated by setting up a link between two nodes in an anechoic room and testing all possible transmission power and attenuation settings. Due to the limited speed at which the LoRa nodes can send and receive packets, the earlier mentioned transmission rate of ten 1-byte data packets per second was achieved through extensive code and timing optimization, thus enabling a full scan of the channel at the four different dynamic range settings every $400 \mathrm{~ms}$, using the aforementioned SF of 7 , a code rate of $4 / 5$ and a bandwidth of $125 \mathrm{kHz}$.

\section{Outdoor trajectory}

In the experiments presented in this work, a medium-build test person moves through a relatively open suburban/urban environment in the south of the city of Ghent, Belgium, while wearing a wireless node both at the front and back of the body. As can be seen in Fig. 5, the trajectory followed by this person starts at the foot of the office building on top of which the base station is located (A). He first walks around a patch of vegetation (B) to the south-west of this tower to gain access to a bridge (C) over the nearby highway, waterway and ring roads. The test person keeps moving north until a distance of $1.5 \mathrm{~km}$ from the base station is reached, where the link is no longer to be considered LoS (between D and E). From this point, the trajectory runs back along the same route. However, when arriving back in the vicinity of the transmitter, a detour (H-I-J) is taken, which runs around several large three- to five-story university buildings before arriving back at the starting point of the trajectory. While moving along this $5.66 \mathrm{~km}$ long route, received SNR values and packet numbers are continuously recorded by both the front and back nodes according to the measurement strategy presented in the previous subsection. First, the test person follows the course on foot, walking at an average speed $\left(v_{R X}\right)$ of $6.2 \mathrm{~km} / \mathrm{h}$. Next, the same course is also traversed using a motorized longboard at an average speed of $31.1 \mathrm{~km} / \mathrm{h}$ to consider the influence of the speed of the mobile receiver on the quality of the links.

\section{Measurement Results And Analysis}

The propagation environment of the trajectory presented in Section II-D is now described and characterized more thoroughly, based of the measurements gathered when walking along this route. Next, the performance gain achieved by applying spatial receiver diversity is investigated, for both selection combining (SC) and maximum ratio combining (MRC), based on the packet reception ratios and the received power level distributions. Finally, the results measured when moving at a higher speed are presented and compared to the channel characteristics when walking.

\section{A. Channel when walking at $6.2 \mathrm{~km} / \mathrm{h}$}

The signal powers measured when walking the trajectory are presented in Fig. 4, showing individual packets as dots and the average received signals, which are calculated by averaging the raw measurements over 9.6 seconds, as lines. Additionally, the average received power is also superimposed on the satellite picture of the trajectory shown in Fig, 5. Upon first observation, there seems to be a large spread of received powers over the entire trajectory. This can mainly be attributed to the movement of the receiver, which causes 
rapidly varying multipath conditions. In general, the trajectory can be split into paths where there is a clear LoS between the base station and the receiver (paths C-D, F-G, H-I and J-A) and areas where there is no LoS (NLoS) (A-C, D-E, G-H and I-J). At the farthest end of the trajectory (D-E), a lot of buildings obstruct the link, resulting in a considerable loss of data packets. Another episode of packet loss can be identified right underneath the base station (A). This is caused by the radiation pattern of the monopole located high above this area in addition to shadowing by the office building underneath it.

1) Path Loss: The path loss $(P L)$ imposed by the propagation environment cannot be characterized for the full dataset due to the complex propagation mechanisms. However, for packets received in LoS areas, an estimation can be made. For two LoS subsets of data, gathered by the front node between markers $F$ and $G$ and by the back node between markers $J$ and A, the path loss characteristics are determined by fitting the data to the conventional formula:

$$
P L(d)=P L\left(d_{0}\right)+10 \cdot n \cdot \log _{10}\left(\frac{d}{d_{0}}\right)
$$

with $d$ denoting the distance from the base station, $n$ being the path loss exponent and $d_{0}=1 \mathrm{~m}$. The path loss exponents found for the selected subsets are $n_{F G}=2.53$ and $n_{J A}=$ 2.64. Path loss exponents are expected to amount to $2.7-3.5$ for urban environments and 3 - 5 in suburban environments [27]. Note that the transmitter was placed very high $(57 \mathrm{~m})$ and very clear LoS paths exist between the base station and the areas between markers F-G and J-A, spanning distances between 150 and $750 \mathrm{~m}$.

2) Front-to-back diversity: When considering the data shown in Fig. 4, it is clear that exploiting a selection combining diversity scheme may significantly increase the average power received by the system, as the fluctuations in the envelopes of the front and back signals are quite complementary. This is especially true for the areas where there is a LoS path between one of the receivers and the base station. In these areas, relatively large signal level differences are caused by body shadowing and the directionality of the wearable antennas.

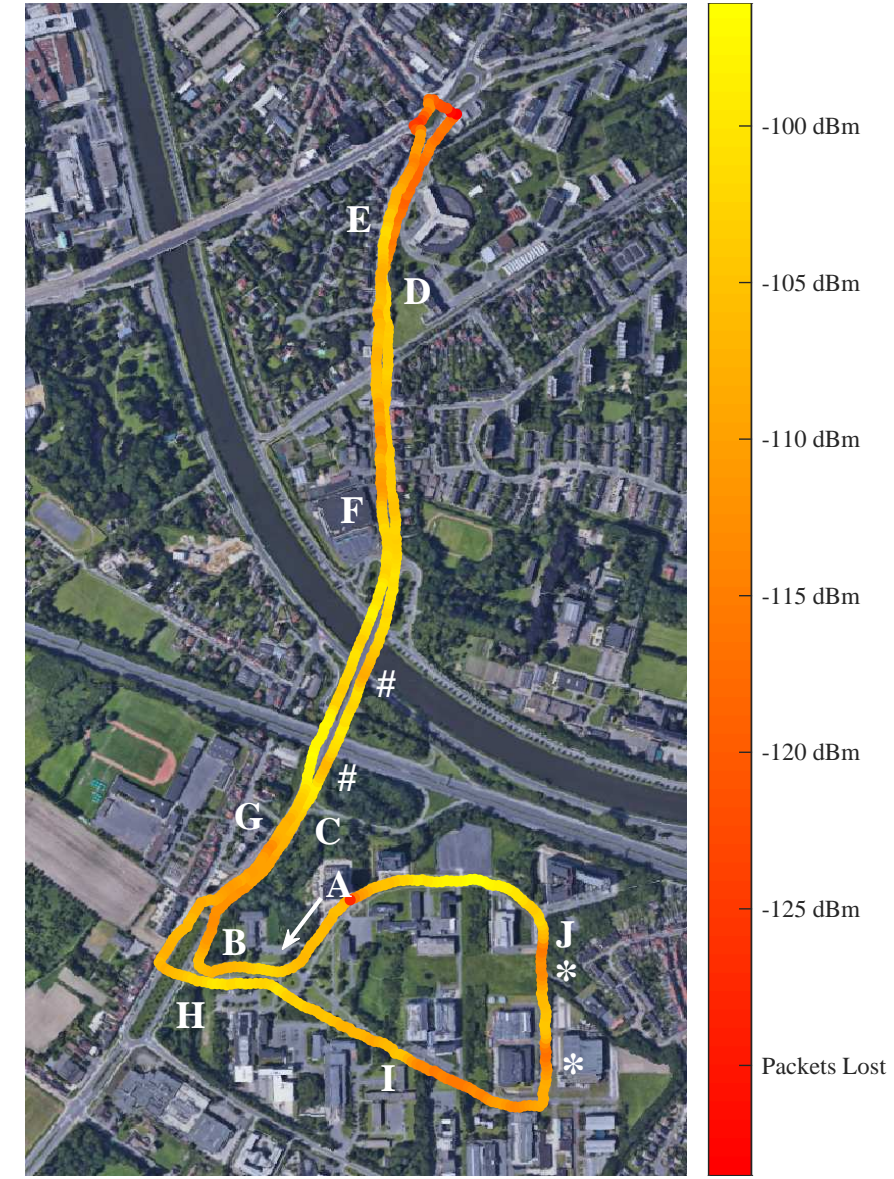

Fig. 5: Full trajectory walked by the test person with indication of the average received signal power measured, using selection combining. $\left(v_{R X}=6.2 \mathrm{~km} / \mathrm{h}\right)$ Map Data: Google, Landsat/Copernicus.

Between markers $\mathrm{E}$ and $\mathrm{F}$, the path is not entirely $\operatorname{LoS}$ due to surrounding houses. Yet, the signals received by the front node are still significantly stronger than those received by the back node. This is mainly caused by a street canyon, in addition to the body shadowing and the antenna directionality mentioned earlier. As a consequence of this behaviour in absence of a true

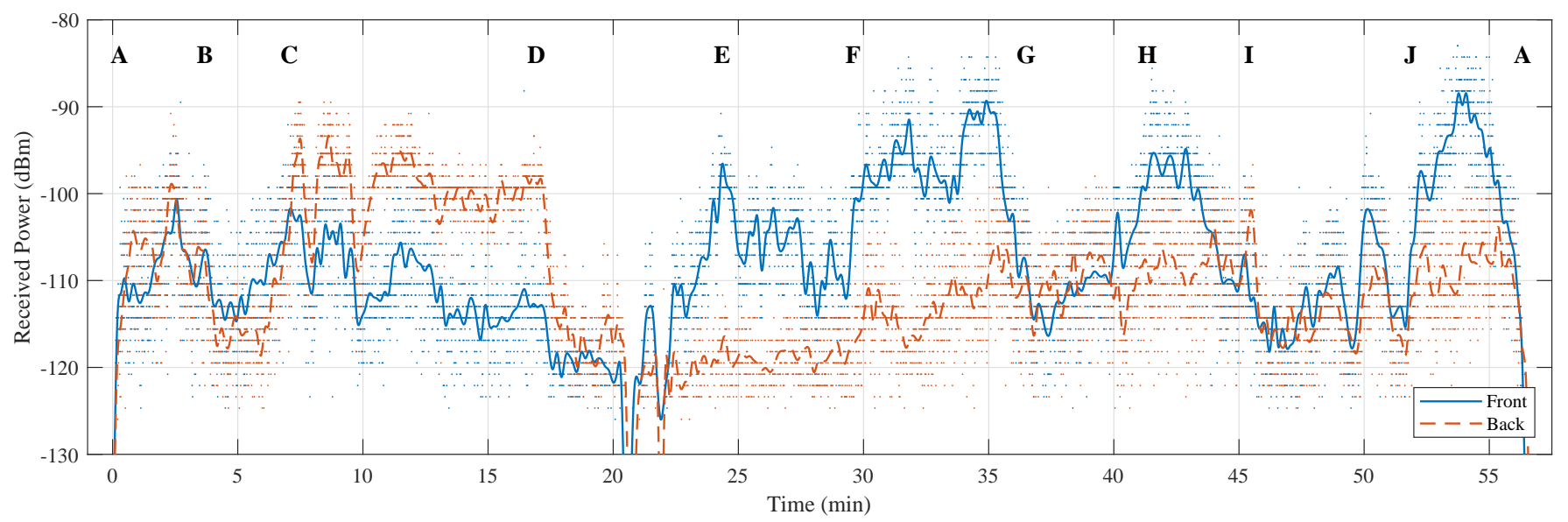

Fig. 4: Average power levels (lines) of all packets (dots) received by both nodes with $v_{R X}=6.2 \mathrm{~km} / \mathrm{h}$. 
line of sight, the wireless link can be described as Quasi-Lineof-Sight (QLoS). Based on the difference between the average signal levels measured in this situation, it can be concluded that selection combining may still solidly improve the average received power.

Also note the shadowing effect caused by the trees north of marker $\mathrm{C}(\#)$, where the connection is otherwise LoS, and between markers $\mathrm{I}$ and $\mathrm{J}(*)$, where the connection is mostly NLoS. In these and other NLoS areas, a selection combiner would not be expected to significantly increase the average signal that is received, as the powers received by the front and back nodes are more comparable.

3) Packet reception ratios: The performance increase of using a selection combining F/B diversity scheme is apparent from the measured packet reception ratios (PRRs), shown in Table III. For all packets sent without any form of attenuation (packet number mod $4=0$ ), the individual PRRs are reasonably high for both body-worn nodes without diversity, which results in a very high PRR of $98.4 \%$ after SC. Moreover, for a transmit power of $-5 \mathrm{dBm}$, an even more significant diversity gain in PRR is observed.

TABLE III: Packet reception ratios for single-receiver and $\mathrm{F} / \mathrm{B}$ receiver diversity situations, evaluated for different transmit powers. $\left(v_{R X}=6.2 \mathrm{~km} / \mathrm{h}\right)$

\begin{tabular}{|c|c|c|}
\cline { 2 - 3 } \multicolumn{1}{c|}{} & \multicolumn{2}{c|}{ PRR (\%) } \\
\cline { 2 - 3 } \multicolumn{1}{c|}{} & $\mathrm{P}_{\mathrm{TX}}=10 \mathrm{dBm}$ & $\mathrm{P}_{\mathrm{TX}}=-5 \mathrm{dBm}$ \\
\hline Front node (SISO) & 92.5 & 54.2 \\
Back node (SISO) & 88.8 & 41.7 \\
SC diversity & 98.4 & 72.9 \\
\hline
\end{tabular}

Table III also reveals a noticeable difference between the PRRs measured at the front and back nodes when considering single-input single-output (SISO) operation. This can be attributed to the asymmetry in the northern part of the trajectory, where most packet loss seems to occur. To obtain more insight into this behaviour and the corresponding PRR variations along this path, the total number of received packets is shown as a function of time in the top half of Fig. 6. The corresponding instantaneous PRRs, measured over a time window of 20 seconds, are shown in the bottom half of Fig. 6. This figure shows that F/B diversity strongly contributes to the packet reception in NLoS situations. Only during two relatively short episodes between the twentieth and twenty-third minute of the experiment, large dips in PRR are registered for both nodes at the same time, resulting in an overall loss of the communication link. These drops in the communication link correspond to two specific parts of the trajectory where the test person first walked through a small tunnel and next crossed the road in an area severely shadowed by the surrounding buildings.

4) Statistics of the received power levels: Table IV shows the average received signal powers together. In general, SC and MRC increase the average received power by some $\mathrm{dB}$ compared to the SISO channels.

Next, cumulative density functions (CDFs) were generated describing the distribution of the received powers w.r.t. the number of packets that were sent. By doing so, the CDFs don't start at 0 because of the packet loss experienced in the wireless

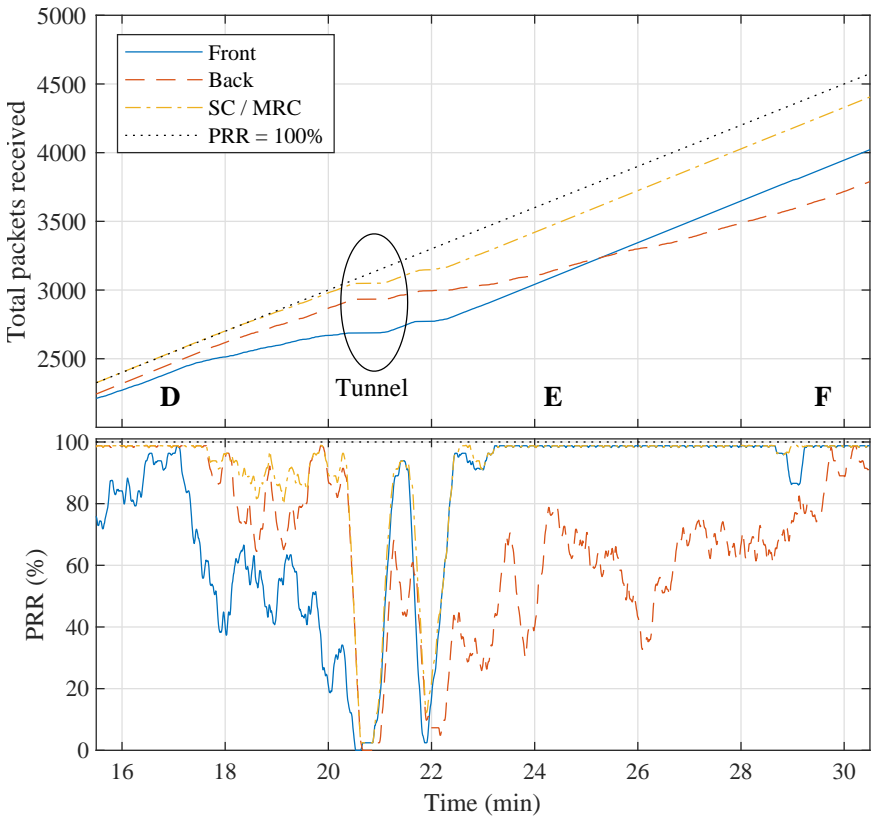

Fig. 6: Total number of received packets (top) and local PRRs (bottom) along the path D-E-F. $\left(v_{R X}=6.2 \mathrm{~km} / \mathrm{h}\right)$

TABLE IV: Average signal levels and corresponding standard deviations. $\left(v_{R X}=6.2 \mathrm{~km} / \mathrm{h}\right)$

\begin{tabular}{|c|c|c|}
\cline { 2 - 3 } \multicolumn{1}{c|}{} & $\mu(\mathrm{dBm})$ & $\sigma(\mathrm{dB})$ \\
\hline Front node (SISO) & -108.7 & 5.9 \\
Back node (SISO) & -111.3 & 5.8 \\
SC diversity & -106.6 & 5.5 \\
MRC diversity & -105.8 & 5.5 \\
\hline
\end{tabular}

links. As a result, Fig. 7 shows both the improvement gained in the distribution of the received power levels as well as the improvement in packet reception. As expected, the distribution for the SC signals are essentially shifted toward higher signal levels when compared to the SISO cases. Additionally, MRC outperforms SC only by a small margin.

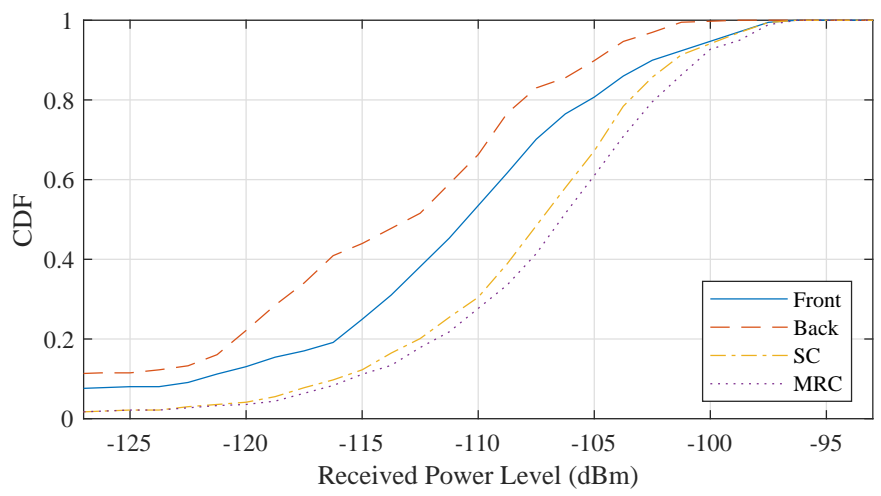

Fig. 7: CDFs of the different links. $\left(v_{R X}=6.2 \mathrm{~km} / \mathrm{h}\right)$

\section{B. Channel when moving at $31.1 \mathrm{~km} / \mathrm{h}$}

Fig. 8 shows the average received power when completing the course with a motorized longboard at $31.1 \mathrm{~km} / \mathrm{h}$. Because of the shorter time-frame, less packets were transmitted, 
lowering the resolution. Generally, the data exhibit the same behaviour as those gathered at lower speed. Because of this similarity, a graphical representation such as shown in Fig. 5 is omitted. The LoS path loss exponent found by considering the area between F-G is equal to 2.44 , which is similar to the previous channel sounding experiment. Due to the movement of the receiver, the amount of data packets was too low to calculate a reliable path loss exponent in the LoS area between markers $\mathbf{J}$ and $\mathrm{A}$.

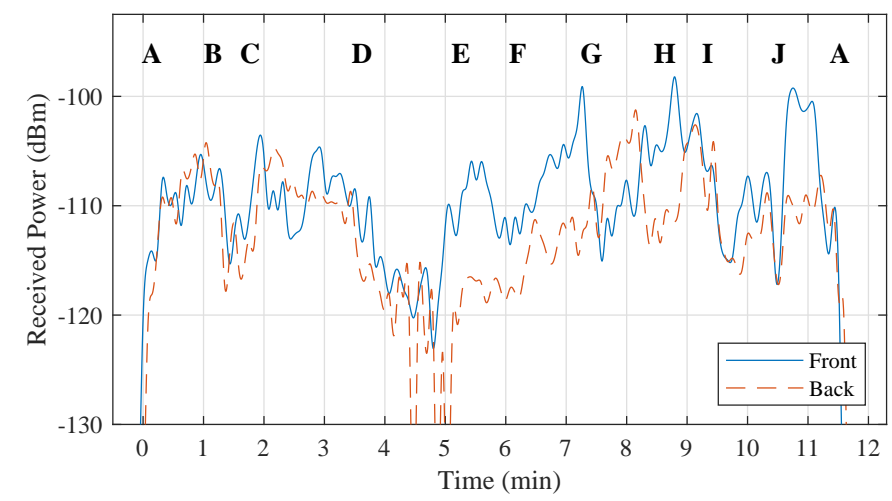

Fig. 8: Average received power with $v_{R X}=31.1 \mathrm{~km} / \mathrm{h}$.

The most significant difference are the lower power levels measured when moving away from the base station between markers $\mathrm{C}$ and $\mathrm{D}$. This is also seen in the overall PRRs, shown in Table V. Overall, the PRR of the back node is $5.5 \%$ lower than the one measured when walking. This is partly made up by the PRR measured by the front node, which is a small $1.2 \%$ higher. The resulting SC/MRC diversity PRR is $1.4 \%$ lower. Furthermore, the PRRs measured when considering a $-5 \mathrm{dBm}$ transmission power are considerably lower, showing respective losses of $8.7 \%, 10.9 \%$ and $13.3 \%$ for front, back and SC/MRC diversity situations. This indicates that at higher speeds, Doppler effects appear to further complicate channel estimation based on packets that are received at a very low SNR.

TABLE V: Packet reception ratios at each individual node and for $\mathrm{F} / \mathrm{B}$ receiver diversity situations at different transmit powers. $\left(v_{R X}=31.1 \mathrm{~km} / \mathrm{h}\right)$

\begin{tabular}{|c|c|c|}
\cline { 2 - 3 } \multicolumn{1}{c|}{} & \multicolumn{2}{c|}{ PRR (\%) } \\
\cline { 2 - 3 } \multicolumn{1}{c|}{} & $\mathrm{P}_{\mathrm{TX}}=10 \mathrm{dBm}$ & $\mathrm{P}_{\mathrm{TX}}=-5 \mathrm{dBm}$ \\
\hline Front node (SISO) & 93.7 & 45.5 \\
Back node (SISO) & 83.3 & 30.8 \\
SC diversity & 97.0 & 59.6 \\
\hline
\end{tabular}

Overall, these higher losses are consistent with the lower average signal levels, shown in Table VI, and the CDF data, shown in Fig. 9. However, the differences between these values and those measured when walking are relatively small and not statistically significant. Additionally, given the great similarity between these data and those gathered during the first measurement campaign presented in this work, this second experiment also serves as a validation for the first one.
TABLE VI: Average signal levels and corresponding standard deviations. $\left(v_{R X}=31.1 \mathrm{~km} / \mathrm{h}\right)$

\begin{tabular}{|c|c|c|}
\cline { 2 - 3 } \multicolumn{1}{c|}{} & $\mu(\mathrm{dBm})$ & $\sigma(\mathrm{dB})$ \\
\hline Front node (SISO) & -109.5 & 4.8 \\
Back node (SISO) & -112.1 & 4.9 \\
SC diversity & -108.4 & 4.9 \\
MRC diversity & -107.5 & 5.0 \\
\hline
\end{tabular}

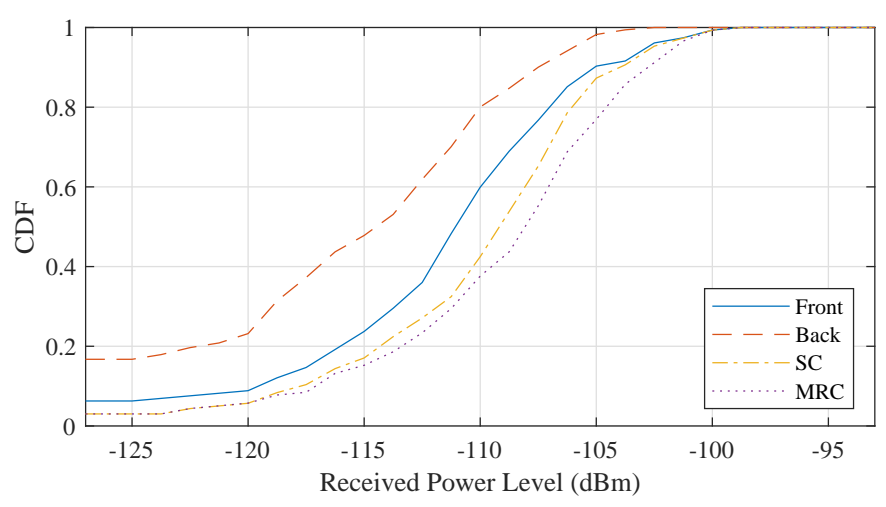

Fig. 9: CDFs of the different links. $\left(v_{R X}=31.1 \mathrm{~km} / \mathrm{h}\right)$

\section{CONCLUSION}

A low-power LoRa link was established between a fixed base station and a mobile user, equipped with a wearable LoRa transceiver node integrated on a substrate-integratedwaveguide antenna, on both the front and back of the body. This setup was calibrated in an anechoic chamber and exploited to perform different measurement campaigns in an omnifarious urban environment, featuring a combination of different line-of-sight (LoS) and non-line-of-sight (NLoS) passages.

By combining the signals received by both receiver nodes, the performance gained by using front-to-back $(\mathrm{F} / \mathrm{B})$ diversity techniques such as selection combining (SC) and maximum ratio combining (MRC) were investigated. SC diversity most notably increased the reliability of the link by significantly increasing the packet reception ratio (PRR) in NLoS areas. This is especially true for packets that were sent at a very low transmit power $(-5 \mathrm{dBm})$, for which increases of $18.7 \%$ and $31.2 \%$ in PRR were observed. MRC was found to provide a marginal additional improvement $(<1 \mathrm{~dB})$ to the system's performance, which does not justify the additional complexity and cost of such a system. However, the comparison of this best-case diversity scenario to single-receiver performance reveals considerable average signal level enhancements up to $5.5 \mathrm{~dB}$.

The trajectory followed when performing the channel sounding experiment was completed once at a steady walking pace of $6.2 \mathrm{~km} / \mathrm{h}$ and a second time moving considerably faster, using a motorized longboard at an average speed of $31.1 \mathrm{~km} / \mathrm{h}$. No statistically significant differences were found between the average signal levels received during both of these runs. However, the data do suggest a slight reduction in PRR and received signal power in certain parts of the trajectory, confirming previous research on the matter. Overall, both measurements do share a great deal of similarity, exhibiting a 
large number of identical features.

In general, the wireless LoRa link is found to be adequately robust, both in terms of range and speed of the user. Given the superior propagation characteristics at sub-GHz frequencies it is proven to be a very valuable choice for low data rate body-centric applications that require a large range and good reliability, especially when combined with F/B diversity.

\section{REFERENCES}

[1] S. Agneessens, "Coupled Eighth-Mode Substrate Integrated Waveguide Antenna: Small and Wideband with High Body-Antenna Isolation," IEEE Access, vol. 6, pp. 1595-1602, 2018. [Online]. Available: http://dx.doi.org/10.1109/ACCESS.2017.2779563

[2] T. Castel, P. Van Torre, L. Vallozzi, M. Marinova, S. Lemey, W. Joseph, C. Oestges, and H. Rogier, "Capacity of Broadband Body-to-Body Channels Between Firefighters Wearing Textile SIW Antennas," IEEE Transactions on Antennas and Propagation, vol. 64, no. 5, pp. 19181931, May 2016.

[3] S. Lemey and H. Rogier, "SIW textile antennas as a novel technology for UWB RFID tags," in 2014 IEEE RFID Technology and Applications Conference (RFID-TA), Sep. 2014, pp. 256-260.

[4] LoRa Alliance. [Online]. Available: https://www.lora-alliance.org/

[5] J. Gaelens, P. Van Torre, J. Verhaevert, and H. Rogier, "LoRa MobileTo-Base-Station Channel Characterization in the Antarctic," Sensors, vol. 17, no. 8, 2017. [Online]. Available: http://www.mdpi.com/1424$8220 / 17 / 8 / 1903$

[6] N. Jovalekic, V. Drndarevic, E. Pietrosemoli, I. Darby, and M. Zennaro, "Experimental Study of LoRa Transmission over Seawater," Sensors, vol. 18, no. 9, 2018. [Online]. Available: http://www.mdpi.com/1424$8220 / 18 / 9 / 2853$

[7] T. Ameloot, P. Van Torre, and H. Rogier, "A Compact Low-Power LoRa IoT Sensor Node with Extended Dynamic Range for Channel Measurements," Sensors, vol. 18, no. 7, p. 2137, Jul 2018. [Online]. Available: http://dx.doi.org/10.3390/s18072137

[8] P. Van Torre, T. Ameloot, and H. Rogier, "Long-range body-to-body LoRa link at $868 \mathrm{MHz}$," in Proceedings of the 13th European Conference on Antennas and Propagation (EuCAP), 2019, pp. 1-5.

[9] M. Shahidul Islam, M. T. Islam, A. F. Almutairi, G. K. Beng, N. Misran, and N. Amin, "Monitoring of the Human Body Signal through the Internet of Things (IoT) Based LoRa Wireless Network System," Applied Sciences, vol. 9, no. 9, p. 1884, May 2019. [Online]. Available: http://dx.doi.org/10.3390/app9091884

[10] F. Wu, J. Redouté, and M. R. Yuce, "WE-Safe: A Self-Powered Wearable IoT Sensor Network for Safety Applications Based on LoRa," IEEE Access, vol. 6, pp. 40846-40853, 2018.

[11] F. Wu, T. Wu, and M. Yuce, "An Internet-of-Things (IoT) Network System for Connected Safety and Health Monitoring Applications," Sensors, vol. 19, no. 1, p. 21, Dec 2018. [Online]. Available: http://dx.doi.org/10.3390/s19010021

[12] J. Petäjäjärvi, K. Mikhaylov, M. Hämäläinen, and J. Iinatti, "Evaluation of LoRa LPWAN technology for remote health and wellbeing monitoring," in 2016 10th International Symposium on Medical Information and Communication Technology (ISMICT), March 2016, pp. 1-5.

[13] J. Petäjäjärvi, K. Mikhaylov, R. Yasmin, M. Hämäläinen, and J. Iinatti, "Evaluation of LoRa LPWAN Technology for Indoor Remote Health and Wellbeing Monitoring," International Journal of Wireless Information Networks, vol. 24, no. 2, pp. 153-165, 62017.

[14] P. A. Catherwood, S. McComb, M. Little, and J. A. D. McLaughlin, "Channel Characterisation for Wearable LoRaWAN Monitors," in Loughborough Antennas Propagation Conference (LAPC 2017), Nov 2017, pp. 1-4.

[15] K. Turbic, L. M. Correia, and M. Beko, "A Channel Model for Polarized Off-Body Communications With Dynamic Users," IEEE Transactions on Antennas and Propagation, vol. 67, no. 11, pp. 7001-7013, Nov 2019.

[16] K. Turbic, S. J. Ambroziak, and L. M. Correia, "A Body-Shadowing Model for Off-Body and Body-to-Body Communications," in 2018 Baltic URSI Symposium (URSI), May 2018, pp. 53-54.

[17] S. L. Cotton, "A Statistical Model for Shadowed Body-Centric Communications Channels: Theory and Validation," IEEE Transactions on Antennas and Propagation, vol. 62, no. 3, pp. 1416-1424, March 2014.
[18] A. Michalopoulou, A. A. Alexandridis, K. Peppas, T. Zervos, F. Lazarakis, K. Dangakis, and D. I. Kaklamani, "Statistical Analysis for On-Body Spatial Diversity Communications at $2.45 \mathrm{GHz}$," IEEE Transactions on Antennas and Propagation, vol. 60, no. 8, pp. 40144019, Aug 2012.

[19] S. L. Cotton and W. G. Scanlon, "Characterization and Modeling of OnBody Spatial Diversity within Indoor Environments at $868 \mathrm{MHz}$," IEEE Transactions on Wireless Communications, vol. 8, no. 1, pp. 176-185, Jan 2009.

[20] M. Hirvonen, C. Böhme, D. Severac, and M. Maman, "On-Body Propagation Performance With Textile Antennas at $867 \mathrm{MHz}$," IEEE Transactions on Antennas and Propagation, vol. 61, no. 4, pp. 21952199, April 2013.

[21] P. Van Torre, L. Vallozzi, L. Jacobs, H. Rogier, M. Moeneclaey, and J. Verhaevert, "Characterization of Measured Indoor Off-Body MIMO Channels with Correlated Fading, Correlated Shadowing and Constant Path Loss," IEEE Transactions on Wireless Communications, vol. 11, no. 2, pp. 712-721, 2012. [Online]. Available: http://dx.doi.org/10.1109/TWC.2011.111611.110298

[22] I. Khan, Y. I. Nechayev, and P. S. Hall, "On-Body Diversity Channel Characterization," IEEE Transactions on Antennas and Propagation, vol. 58, no. 2, pp. 573-580, Feb 2010.

[23] S. Wiszniewski and S. J. Ambroziak, "Characterization of Slow and Fast Fading in Off-Body Communication at $2.45 \mathrm{GHz}$ with Space Diversity Scheme in an Indoor Environment," International Journal of Antennas and Propagation, 2019.

[24] U. Noreen, A. Bounceur, and L. Clavier, "A Study of LoRa Low Power and Wide Area Network Technology," in 2017 International Conference on Advanced Technologies for Signal and Image Processing (ATSIP), May 2017, pp. 1-6.

[25] J. C. Liando, A. Gamage, A. W. Tengourtius, and M. Li, "Known and Unknown Facts of LoRa: Experiences from a Large-scale Measurement Study," ACM Trans. Sen. Netw., vol. 15, no. 2, pp. 16:1-16:35, Feb. 2019. [Online]. Available: http://doi.acm.org/10.1145/3293534

[26] J. Petäjäjärvi, K. Mikhaylov, M. Pettissalo, J. Janhunen, and J. Iinatti, "Performance of a Low-Power Wide-Area Network Based on LoRa Technology: Doppler Robustness, Scalability, and Coverage," International Journal of Distributed Sensor Networks, vol. 13, no. 3, 2017. [Online]. Available: https://doi.org/10.1177/1550147717699412

[27] T. S. Rappaport, Wireless Communications: Principles and Practice. Upper Saddle River, New Jersey: Prentice Hall PTR, 1996.

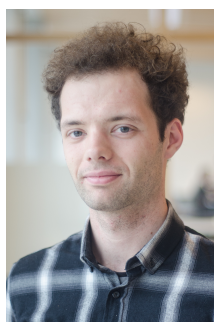

Thomas Ameloot was born in 1995. He received the B.Sc. and M.Sc. degrees in electronics and ICT engineering technology from Ghent University, Ghent, Belgium, in 2016 and 2017, respectively, where he is currently pursuing the Ph.D. degree in electrical engineering with the Department of Information Technology. His current research efforts are primarily concerned with the physical performance of long-range, low-power wireless communication technologies. 


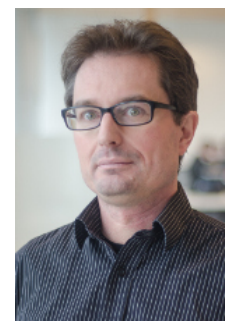

Patrick Van Torre received the M.Sc. degree in Electrical Engineering from I.H. BME-CTL in Ghent, Belgium in 1995 and the Ph.D. degree in Electrical Engineering from Ghent University in 2012. After working for a number of years as a hardware and software designer in industry, he started as a lecturer at University College Gent, Belgium in 1999. There, he was a pioneer in setting up project-oriented lab courses in a variety of different topics, ranging from analog electronics to digital signal processing and embedded systems. In 2011, his project concerning the development of didactical equipment was awarded the Queen Paola Award in Education. In combination with his ample educational responsibilities, Patrick Van Torre started his first research activities in 2008 and obtained a Ph.D. degree from Ghent University, Belgium in 2012, including 6 journal and 7 conference proceedings papers as well as a scientific award.

He is currently an Assistant Professor with the Department of Information Technology, Ghent University, and a Guest Professor with imec. He is responsible for multiple courses in Electronics and ICT and is active as a researcher, in the field of analog electronics and wireless communications. He has authored or co-authored over 100 publications in international journals and in conference proceedings. His current research interests include bodycentric multiple-input multiple-output (MIMO) and beam-forming systems as well as on software defined radio and sub-GHz long range wireless sensor networks.

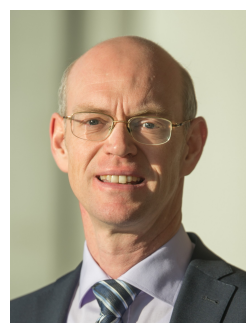

Hendrik Rogier (SM'06) received the M.Sc. and Ph.D. degrees in electrical engineering from Ghent University, Ghent, Belgium, in 1994 and 1999, respectively. From 2003 to 2004, he was a Visiting Scientist with the Mobile Communications Group, Vienna University of Technology, Vienna, Austria. $\mathrm{He}$ is a currently a Full Professor with the Department of Information Technology, Ghent University, a Guest Professor with the Interuniversity Microelectronics Centre, Ghent, and a Visiting Professor with the University of Buckingham, Buckingham, U.K. He has authored or co-authored over 170 papers in international journals and over 190 contributions in conference proceedings. His current research interests include antenna systems, radio wave propagation, body-centric communication, numerical electromagnetics, electromagnetic compatibility, and power/signal integrity.

Dr. Rogier is a member of Technical Committee 24 on RFID Technology with the IEEE Microwave Theory and Techniques Society (MTT-S) and a member of the Governing Board of Topical Group MAGEO on Microwaves in Agriculture, Environment and Earth Observation with the European Microwave Association, Leuven, Belgium. He was a recipient of the URSI Young Scientist Award (twice) at the 2001 URSI Symposium on Electromagnetic Theory and at the 2002 URSI General Assembly, the 2014 Premium Award for Best Paper in the IET Electronics Letters, the Best Paper Award First Place in the 2016 IEEE MTT-S Topical Conference on Wireless Sensors and Sensor Networks, the Best Poster Paper Award at the 2012 IEEE Electrical Design of Advanced Packaging and Systems Symposium, the Best Paper Award at the 2013 IEEE Workshop on Signal and Power Integrity, and the Joseph Morrissey Memorial Award for the First Best Scientific Paper at BioEM 2013. From 2017 until 2019, he was an Associate Editor of IEEE Transactions on Microwave Theory and Techniques. Currently, he is an Associate Editor of IET Electronics Letters and of IET Microwaves, Antennas and Propagation. $\mathrm{He}$ acts as the URSI Commission B representative for Belgium. 